\title{
Salpingitis Due to Entamoeba histolytica
}

Calore E.E., Calore N.M.P. and Cavaliere M.J.
Emílio Ribas Institute, São Paulo, SP; Adolfo Lutz

Institute, São Paulo, SP, Brazil

\begin{abstract}
We describe the pathology of a unique case of Fallopian tube amebiasis, associated with hydrosalpinx, in a 21-year-old woman. She complained of lower abdominal pain, had a foul-smelling green vaginal discharge and fever during one week. There was a discrete increase in body temperature and a painful abdominal palpation at the lower right side, with signs of local peritoneal irritation. Pathological examination showed a marked dilatation of the fallopian tube and hydrosalpinx. Microscopic examination showed a poorly formed granuloma composed of large macrophages with many Entamoeba histolytica trophozoites inside the fallopian tube. Even though it is a rare disease the correct diagnosis of female genital tract amebiasis is of great importance for the indication of proper therapy.

Key Words: Female genital tract, amebiasis, parasites, fallopian tube, female genital tract infection, salpingitis.
\end{abstract}

The female genital tract can harbor a variety of viral, bacterial, fungal and parasitic organisms. Bacterial cervicitis is the most common cause of these female genital tract infections, followed by viral and fungal cervicitis. Ovarian and fallopian tube infectious diseases are less frequent and are usually caused by an ascendant infection through the cervix or they represent a manifestation of transmitted pelvic inflammatory disease. Granulomatous salpingitis can be provoked by various kinds of organisms. Tuberculosis, actinomycosis, schistosomiasis, hydatic disease and pinworm can be etiologies for granulomatous salpingitis.

Only rare reports of upper female genital tract amebiasis have been described. Gogoi (1969) reported two women with a blood-stained vaginal discharge and vulvar or vaginal ulcers that proved to

Received on 5 August 2001; revised 27 February 2002.

Address for correspondence: Dr. Edenilson Eduardo Calore MD, Ph.D. Emílio Ribas Institute - Section of Pathology. Av. Dr. Arnaldo, 165 - 01246-001 - São Paulo, Brazil. Fax: (55 11)8437485.E-mail: calore@uol.com.br

The Brazilian Journal of Infectious Diseases 2002;6(2):97-99 (C) 2002 by The Brazilian Journal of Infectious Diseases and Contexto Publishing. All rights reserved.

$1413-8670$ be caused by Entamoeba histolytica. Three cases of cytological detection of Entamoeba histolytica infection of the female genital tract were reported by Kapila and Verma (1982). Haibach et al (1985) described the association of an Entamoeba histolytica infection in a 69-year-old woman who had a well-differentiated squamous cell carcinoma of the uterine cervix five years after this cervical infection. Nopdonrattakoon (1996) described a case of a 56year-old woman with multiple small ulcerated and friable lesions with contact bleeding of the vulva, vagina and cervix; wet smears showed typical Entamoeba histolytica trophozoites.

We describe a case of fallopian tube amebiasis due to Entamoeba histolytica in a 21-year-oldwoman whose diagnosis was not suspected before the histological study.

\section{Case report}

We received the right fallopian tube of a 21-yearold-woman. She complained of lower abdominal pain, and had a foul-smelling green vaginal discharge and fever during one week. She reported no diarrhea or constipation and reported sexual intercourse with 
various partners. This patient had a discrete increase in body temperature and a painful abdominal palpation with signs of local peritoneal irritation. An intense inflammatory smear was observed on cytological examination, but no specific agent was detected. She was submitted to surgery. There were no intestinal lesions nor was the bowel perforated. Macroscopic examination showed a hydrosalpinx, with multiple stenosis, that completely distorted the normal structure of the tube. Histological studies revealed a mild inflammatory mononuclear process composed of macrophages, lymphocytes and plasma cells in the mucosa of the fallopian tube. A poorly formed granuloma composed of many large macrophages and some lymphocytes was observed in the distal portion of the fallopian tube wall and serosal coat. Many 15 to $25 \mu \mathrm{m}$ roughly rounded or ovoid ameboid structures were observed by H\&E staining inside these macrophages. These structures had spherical and generally eccentric nuclei with an easily recognized karyosome, corresponding to Entamoeba histolytica trophozoites (Figures 1 and 2 ). The trophozoites were strongly PAS positive. Isolated macrophages containing the amebic trophozoites were also observed in the mucosal layer. The right ovary was substituted by fibrovascular tissue. Immunohistochemical studies (Hsu et al., 1981) showed that the inflammatory process was formed by a large number of CD-68 positive macrophages, a moderate number of $\mathrm{T}$ lymphocytes (CD-45RO cells) distributed at random among the macrophages, and rare B cells (CD-20).

\section{Discussion}

The morphology of the parasite observed in this study was characteristic of Entamoeba histolytica. This pathogen generally causes gastrointestinal infection and sometimes attacks other organs, such as the brain, liver, spleen and lungs. Involvement of the genitourinary tract has only rarely been reported. The rarity of female genital tract amebiasis may be due to the high vaginal acidity.
The immunohistochemical studies demonstrated predominance of a cellular immune response with a large number of macrophages in the granuloma. Most of the trophozoites were inside these macrophages. The $\mathrm{T}$ cell response was moderate and was distributed at random among the macrophages. These cells have a key role in the cellular response since they contribute to the attraction and immobilization of the macrophages. The B cell's contents were scarce in the inflammatory reaction.

Most cases of female genital infections due to $E$. histolytica present cervical discharge and vulvar, vaginal or cervical ulcers. Sometimes the macroscopic aspect on speculum examination reveals malignancy, as in two cases described by Kapila and Verma (1982) and as in the case we describe here. Therefore, even though it is a rare disease correct diagnosis is of great importance for proper therapy and to avoid unnecessary surgical procedures.

\section{References}

1. Gogoi M.P. Amebiasis of the female genital tract. Am J Obstet Gynecol 1969;105(8):1281-2.

2. Haibach H., Bickel J.T., Podrecca G.I., Llorens A.S. Squamous cell carcinoma of the uterine cervix subsequent to amebiasis. Arch Pathol Lab Med 1985;109(12):1121-3.

3. Heinz K.P. Amoebic infection of the female genital tract. A report of three cases. S Afr Med J 1973;47(39):1795-8.

4. Hsu S.M., Raine L., Fanger H. The use of antiavidin antibody and avidin-biotin peroxidase complex in immunoperoxidase techniques. Am J Clin Pathol 1981;75: 816-20.

5. Kapila K., Verma K. Cytologic detection of parasitic disorders. Acta Cytol 1982;26(3):359-62.

6. Nopdonrattakoon L. Amoebiasis of the female genital tract: a case report. J Obstet Gynaecol Res 1996;22(3): 235-8. 
Figure 1. A spherical structure with an eccentric nucleus in which a karyosome can be easily recognized, which corresponds to a trophozoite of Entamoeba histolytica (arrow) (x 900)

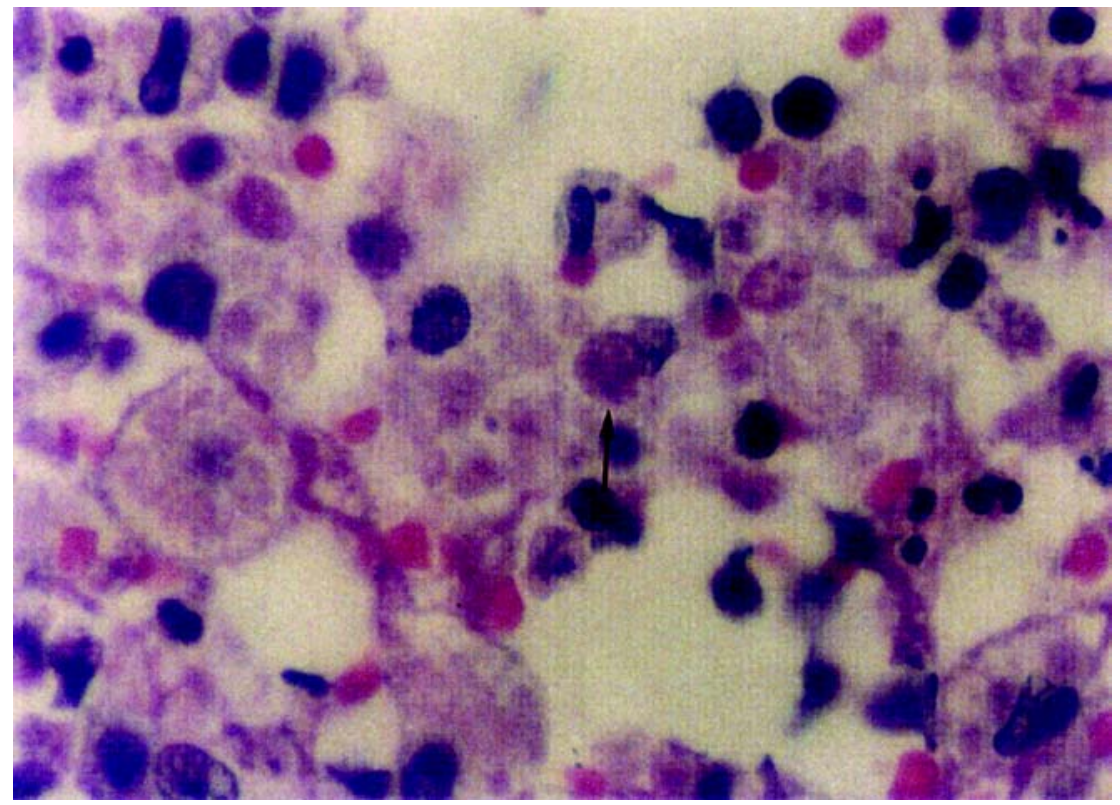

Figure 2. In this figure many trophozoites can be seen inside macrophages (arrow) (x 900)

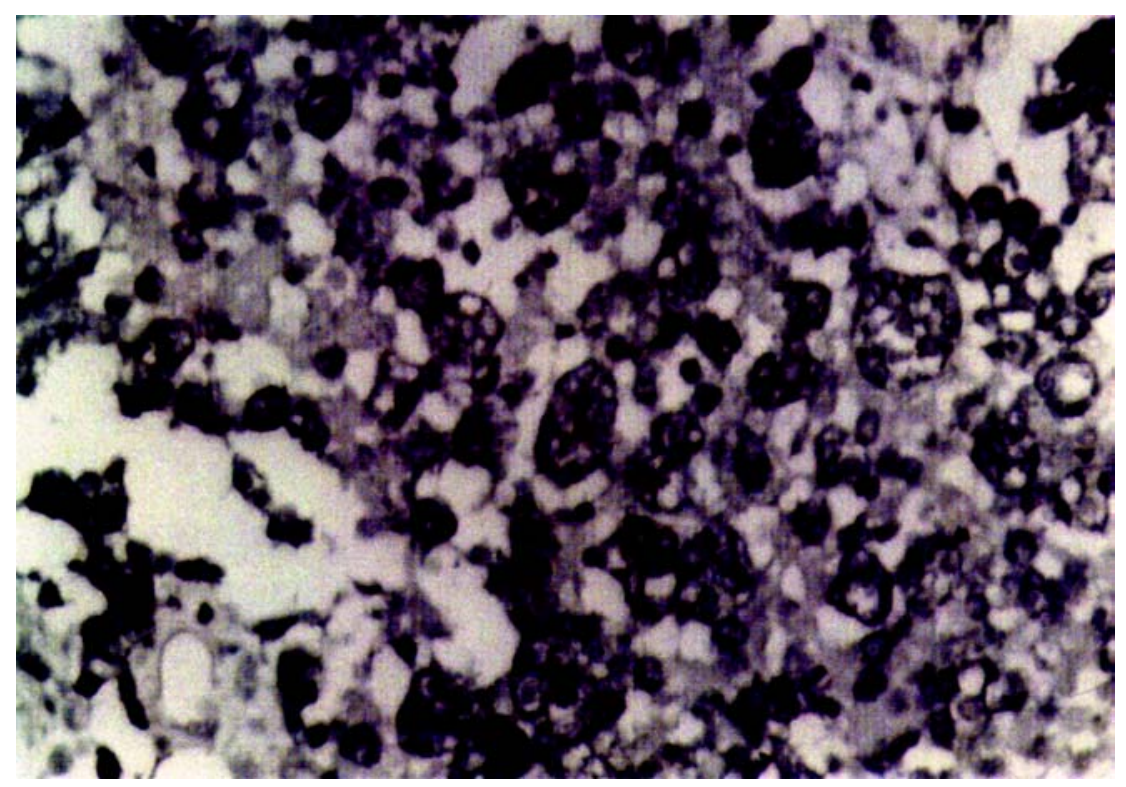

